\title{
Variation in the dust spectral index across M33
}

\author{
Fatemeh S. Tabatabaei ${ }^{1}$, Jonathan Braine ${ }^{2}$, Carsten Kramer ${ }^{3}$, \\ Manolis Xilouris ${ }^{4}$, Mederic Boquien ${ }^{5}$, Simon Verley ${ }^{6}$, \\ Eva Schinnerer ${ }^{1}$, Daniela Calzetti ${ }^{7}$, Francoise Combes $^{8}$, Frank Israel $^{9}$, \\ Christian Henkel ${ }^{10}$ and The HerM33es Team \\ ${ }^{1}$ Max-Planck-Institut für Astronomie, Königstuhl 17, 69117-Heidelberg, Germany \\ ${ }^{2}$ Laboratoire d'Astrophysique de Bordeaux, Université de Bordeaux, 33271 Floirac, France \\ ${ }^{3}$ Instituto Radioastronomia Milimetrica, 18012 Granada, Spain \\ ${ }^{4}$ Institute of Astronomy and Astrophysics, National Observatory of Athens, GR-15236 Athens, \\ Greece \\ ${ }^{5}$ Laboratoire d'Astrophysique de Marseille, UMR 6110 CNRS, Marseille, France \\ ${ }^{6}$ Department Física Teórica y del Cosmos, Universidad de Granada, Spain \\ ${ }^{7}$ Department of Astronomy, University of Massachusetts, Amherst, MA 01003, USA \\ ${ }^{8}$ Observatoire de Paris, 75014 Paris, France \\ ${ }^{9}$ Sterrewacht Leiden, Leiden University, PO Box 9513, 2300 RA, Leiden, The Netherlands \\ ${ }^{10}$ Max-Planck-Institut für Radioastronomie, Auf dem Hügel 69, 53121-Bonn, Germany \\ email: taba@mpia.de
}

\begin{abstract}
Using the Herschel PACS and SPIRE FIR/submm data, we investigate variations in the dust spectral index $\beta$ in the nearby spiral galaxy M33 at a linear resolution of $160 \mathrm{pc}$. We use an iteration method in two different approaches, single and two-component modified black body models. In both approaches, $\beta$ is higher in the central disk than in the outer disk similar to the dust temperature. There is a positive correlation between $\beta$ and $\mathrm{H} \alpha$ as well as with the molecular gas traced by $\mathrm{CO}(2-1)$. A Monte-Carlo simulation shows that the physical parameters are better constrained when using the two-component model.
\end{abstract}

Keywords. Interstellar Medium, Star formation, Dust, Galaxies: M33

\section{Introduction}

Inferring the nature of dust and dust heating requires knowledge about the dust emissivity, and its power law index. There is some evidence supporting variation of dust emissivity with environmental conditions (e.g. Lisenfeld et al. 2000, Paradis et al. 2009). The dust spectral index could change depending on grain properties, e.g. geometry, size distribution, and chemical composition. Dust grains might be affected by different physical processes like shattering, sputtering, grain - grain collision, condensation and coagulation. Hence, depending on recent star formation activities, dust/gas content and metallicity changes across galaxies, variations in the dust spectral index in inner/outer disks or arm/inter-arm regions may occur. Herschel observations of the nearest Scd galaxy M33, (HerM33es, Kramer et al. 2010, Boquien et al. 2010) ideally enable us to derive distribution of $\beta$ across M33.

We use the Herschel PACS \& SPIRE data at 160, 250, 350, and $500 \mu \mathrm{m}$ together with the Spitzer MIPS data at $70 \mu \mathrm{m}$ to investigate the physical properties of a standard dust model (Big grains+vsg+PAH, Krügel 2003 and references therein). These wavelengths 

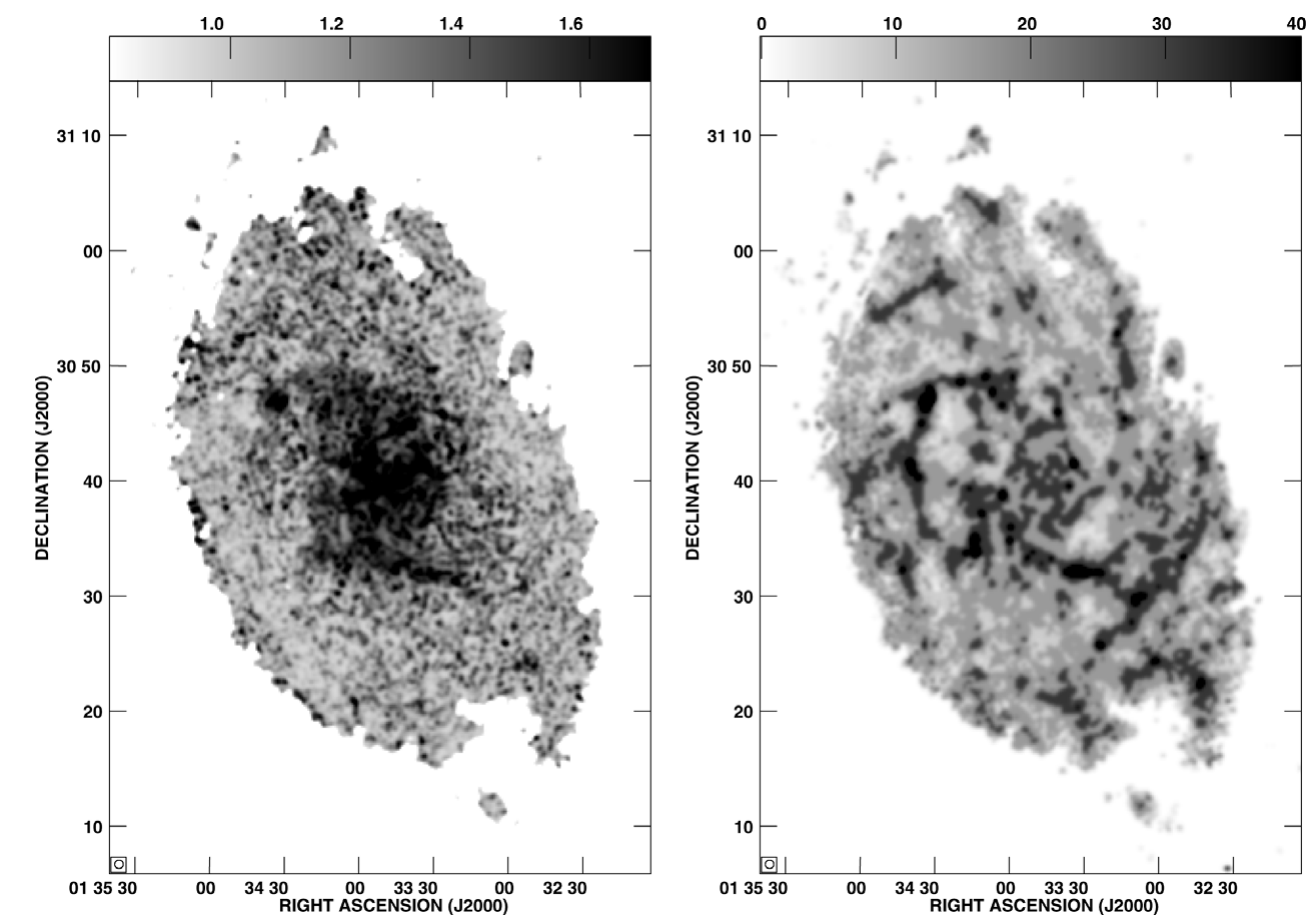

Figure 1. Distribution of dust spectral index (right) and column density (in $\mu \mathrm{g} \mathrm{cm}^{-2}$, right) across M33, based on the single-component approach.

spread over a range where the dust emission mainly emerges from the big grains, which are believed to be in thermal equilibrium with the interstellar radiation field.

\section{Analysis and Results}

We use the Newton-Raphson iteration method to derive T, $\beta$ and column density, pixel by pixel, in two different approaches:

1) We assume that dust emitting at the SPIRE bands is mainly heated by the interstellar radiation field (ISRF) and obeys single - component gray body radiation. In this case we use the data at longer wavelengths $(250,350$ and $500 \mu \mathrm{m})$.

2) We consider another dust component heated by young massive stars with higher temperature (warm dust) besides the dust heated by the ISRF (cold dust), hence, dust emits as a two-component gray body. Here, we use the data from $70 \mu \mathrm{m}$ to $500 \mu \mathrm{m}$ wavelengths. Using a Monte-Carlo simulation, we further investigate the reliability of each approach and estimate the errors and uncertainties. We derive the following results:

Single Component Approach As shown in Fig. 1, in the central disk $(R<4 \mathrm{kpc})$, the dust spectral index is higher in the central disk $(\beta>1.4)$ than in the outer disk. Similarly, both $\mathrm{T}$ and column density are higher in the central disk. The flocculent spiral structure of M33 is evident in the column density map. The average $\mathrm{T}$ and $\beta$ for the entire galaxy are $22 \pm 9 \mathrm{~K}$ and $1.3 \pm 0.5$, respectively.

Two Component Approach The cold and warm dust components are separated assuming a variable $\beta$ for the dominant colder component. Although we have to use a fixed value of $\beta_{\mathrm{warm}}$ for the warm dust, we repeat the analysis for different possibilities of $\beta_{\mathrm{warm}}=1,1.5$, and 2 . Interestingly, we find that $\beta$ is again higher in the central 

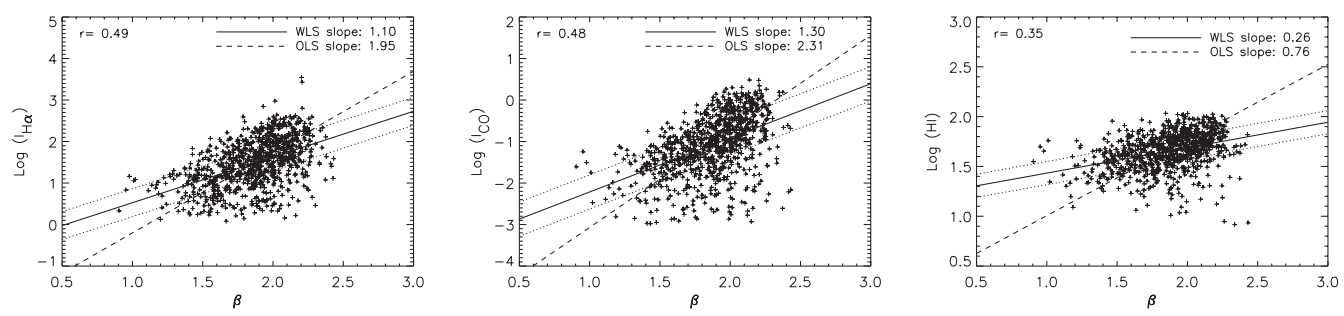

Figure 2. Correlations between the dust spectral index and $\mathrm{H} \alpha, \mathrm{CO}(2-1)$, and $\mathrm{HI}$ integrated intensities. The WLS weighted least square fit (solid line) and its 5-sigma confidence levels (dotted lines) are shown. The dashed lines show the ordinary least square fit (OLS).

disk and even shows the arm/inter-arm contrast, irrespective of $\beta_{\mathrm{warm}}$ assumed. Also in all three cases, the cold dust follows spiral arms and dust lanes and is denser where the temperature is lower. The warm dust (both temperature and column density) follows the distribution of $\mathrm{H} \alpha$ and star forming regions. For the entire galaxy, we derive $\mathrm{T}_{\text {cold }}=16 \pm 3 \mathrm{~K}, \mathrm{~T}_{\text {warm }}=40 \pm 13 \mathrm{~K}$, and $\beta=1.7 \pm 0.4$ assuming $\beta_{\text {warm }}=2$. Our simulations show that the two-component approach, generally, constrains the parameters better than the single-component approach, particularly the temperature.

\section{Correlation with ISM Tracers}

The M33's outer/inner disk and even the spiral arms are visible in the $\beta$-maps. Having similar distribution as of the molecular gas and radiation field traced by $\mathrm{H} \alpha, \beta$ seems to have a meaningful change across the galaxy. Figure 2 shows correlations between $\beta$ and $\mathrm{H} \alpha, \mathrm{CO}(2-1)$ and $\mathrm{HI}$ intensities (Gratier et al. 2010) as tracers of the ionized, molecular, and atomic phases of the ISM, respectively. In both approaches, $\beta$ is found to be better correlated with both $\mathrm{H} \alpha$ and $\mathrm{CO}$ than with $\mathrm{HI}$. This could indicate that $\beta$ is not the same in star forming and non-starforming regions in a galaxy. Star formation could in principle influence the grain composition (via a change in metallicity) and size distribution (via shattering of dust grains due to strong Shocks), which could modify the dust emissivity index i.e. $\beta$.

\section{References}

Boquien, M., Calzetti, D., Kramer, C. et al. 2010, A\&SA, 518, 70

Gratier P., Braine, J., Rodriguez-Fernandez, N. J. et al. 2010, A\& A, 522, 3

Kramer, C., Buchbender, C., Xilouris, E. M. et al. 2010, A\&A, 518, 67

Krügel, E. 2003, The Physics of Interstellar Dust (IoP Series in astronomy and astrophysics, ISBN 0750308613)

Lisenfeld, U., Isaak, K. G., \& Hills, R. 2000, MNRAS, 312, 433

Paradis, D., Bernard, J.-Ph., \& Mény, C. 2009, A\&A, 506, 745 